\title{
Medizin aus Leidenschaft
}

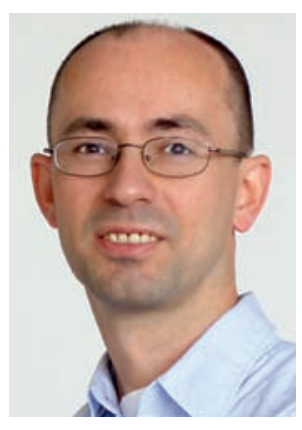

Dr. med. Dieter Schmid, Redaktionsleitung
„Also ich könnte das nicht!“ Das hören wir Mediziner oft aus unserem Umfeld. Sich Tag für Tag mit den Leiden anderer Menschen herumschlagen? Lange Schichtdienste? Extreme Verantwortung? Ja, offensichtlich sind Mediziner eben doch ein ganz spezieller Menschenschlag. Verschärft gilt das für den Lieblingsfacharzt der TV-Serien-Macher: den Rechtsmediziner. Wenn Protagonisten dieses Faches wie Prof. Boerne im Münsteraner Tatort oder Dr. Robbins bei CSI halb verweste Leichen aufschneiden, um der Todesursache auf die Spur zu kommen, sitzen „normale“ Menschen vor dem Fernseher, schütteln den Kopf und rufen laut: „Wie können die nur!“Junge Mediziner schauen sich das an und sagen: „Das will ich auch!“

In diesem Heft ist die Rechtsmedizin unser Top-Thema. Vier Rechtsmediziner erzählen von ihrer Leidenschaft für dieses Fach - und erläutern die Vor- und Nachteile. Dabei wird rasch klar: Rechtsmediziner klären in Zusammenarbeit mit Polizei und Staatsanwaltschaft zwar tatsächlich viele Tötungsdelikte auf. Die wahre Aufgabenpalette ist aber viel breiter. Zum ganzen Bild gehört, dass Rechtsmediziner natürlich auch mit Lebenden arbeiten. So helfen sie zum Beispiel durch den Nachweis von Missbrauch, Kinder vor Gewalttätern zu schützen. Mehr lesen Sie in unserem Top-Artikel auf Seite 16.

Das ganze Bild der Medizin darstellen, weit über den Tellerrand des Studiums hinaus - das liegt uns bei Via medici seit 18 Jahren am Herzen. An dieser Stelle möchte ich mich bei Ihnen herzlich bedanken für all die Kommentare, das Feedback, die Unterstützung und Ihre Begeisterung für die Via medici. Es macht uns allen unglaublich viel Spaß, für Sie zu recherchieren und zu schreiben. 2013 wird ein spannendes Jahr, und es wird sich einiges ändern bei Via medici. Als Erstes werden wir günstiger! Statt $24,95 €$ wird das Studenten-Abo nun nur noch 19,95€ kosten, und die Versandkosten fallen ganz weg. Damit aber nicht genug. Die Via medici wird nun sechsmal im Jahr erscheinen. Bleiben Sie gespannt - wir haben noch mehr für Sie!

Ich wünsche Ihnen viel Freude mit dieser Via medici-Ausgabe - und Durchhaltekraft für die verbleibenden Wintermonate. Der Sommer kommt!

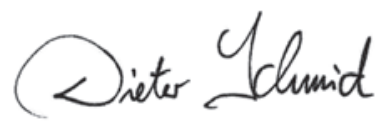

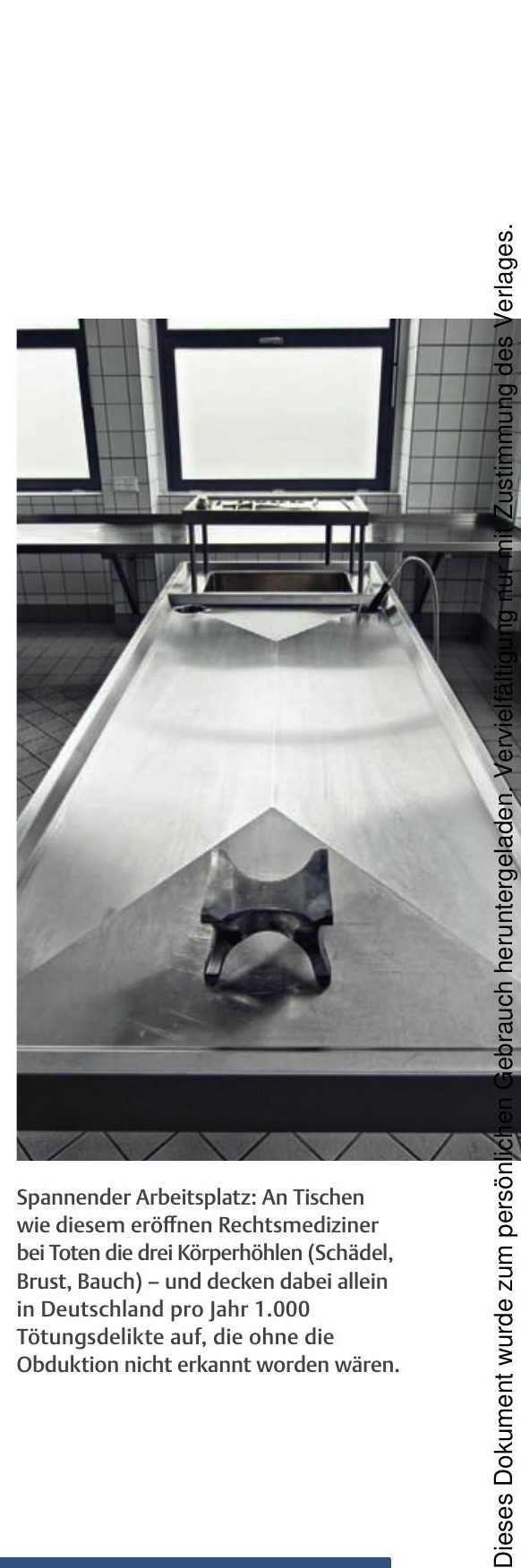

ONLINE-UMFRAGE

Produkte statt Patienten: Lust auf eine Karriere in der Industrie?

Wer Medizin studiert, wird Arzt? Nicht unbedingt! Auch in der freien Wirtschaft werden Mediziner gesucht - und viele junge Ärzte folgen dem Ruf. Schließlich bietet die Industrie nicht nur humanere Arbeitszeiten, sondern auch äußerst vielseitige Aufgaben. Die vier Mediziner, die in diesem Heft auf $\mathbf{S} . \mathbf{2 4}$ erzählen, wie sie den Sprung in die Industrie schafften, sind jedenfalls überzeugt, das Richtige getan zu haben. Einige von ihnen haben zunächst ein paar Jahre in der Klinik gearbeitet.
Andere haben gleich im Studium gemerkt, dass der Patientenkontakt sie nicht so ganz ausfüllt. Wie ist das bei Ihnen? Möchten Sie unbedingt praktizieren, oder könnten Sie sich auch vorstellen, bei der Entwicklung von Industrieprodukten (egal welcher Art) mitzuwirken?

\section{Wäre ein Arbeitsplatz in der Industrie eine reelle Berufsoption für Sie?}

Geben Sie Ihr Statement ab und kommentieren Sie unter: http://bit.ly/UVFoHI
- Ergebnis der Umfrage in Via medici 5.12: Wir fragten, ob eine Fernbeziehung parallel zum Medizinstudium funktionieren kann. Die Teilnehmer sind sich einig: Wahre Liebe überwindet alle Hindernisse. Eine Teilnehmerin trennen 1.400 km von ihrem Liebsten - sie studiert in Budapest, er in Köln. Trotzdem hält die Fernbeziehung, und seit August 2011 führen die beiden sogar eine „Fern-Ehe“. Sie schreibt: „Es ist sehr zeit- und arbeitsintensiv, eine Beziehung über eine so große Distanz zu führen, aber diese Erfahrung hat uns beide in unserer Liebe gestärkt! “ Mehr Kommentare unter: .../aktuelles/aktion/miniumfrage5-12.html 